\title{
Outcome of a Rare Case of Fetal Solitary Cardiac Rhabdomyoma not Associated with Tuberous Sclerosis
}

\author{
${ }^{1}$ Nageshu Shailaja, ${ }^{2}$ Kulkarni Namrata, ${ }^{3}$ Lingegowda Krishna, ${ }^{4}$ Vanishree, ${ }^{5}$ BS Bhat, ${ }^{6}$ Krishna Kirtan, ${ }^{7}$ Gunda Swathi
}

\begin{abstract}
At 19 weeks 6 days ultrasonography of a 31-year-old G2P1L1 revealed a small echogenic area seen in the subvalvular apparatus of the mitral valve (anterior mitral leaflet) suggestive of cardiac rhabdomyoma (CR). At 28 weeks of gestation there was mild increase in the size of the $\mathrm{CR}(13 \times 6 \mathrm{~mm})$. There was no evidence of hemodynamic compromise. The interval growth of the fetus was satisfactory. The family tree of three generations was examined and revealed no evidence of Tuberous Sclerosis. She delivered a live 34 weeks $2.2 \mathrm{~kg}$ male baby with 8 and 9 Apgar scores at 5 and 10 minutes respectively. Baby suddenly developed cyanosis and died 24 hours after birth. The fetal autopsy confirmed the diagnosis of CR. Genetic analysis of neonatal blood revealed no evidence of TSC mutation suggestive of tuberous sclerosis.
\end{abstract}

Keywords: Cardiac rhabdomyoma, Tuberous sclerosis complex, Antenatal diagnosis.

How to cite this article: Shailaja N, Namrata K, Krishna L, Vanishree, Bhat BS, Kirtan K, Swathi G. Outcome of a Rare Case of Fetal Solitary Cardiac Rhabdomyoma not Associated with Tuberous Sclerosis. Donald School J Ultrasound Obstet Gynecol 2014;8(2):226-228.

Source of support: Nil

Conflict of interest: None

\section{INTRODUCTION}

The vast majority of primary cardiac tumors in children are benign, whilst approximately $10 \%$ are malignant. Secondary malignant tumors are 10 to 20 times more prevalent than primary malignant tumors. The incidence of primary cardiac tumors varies between 0.0017 and $0.027 \%$ of all live births. Most are rhabdomyomas $(60 \%)$, teratomas $(20 \%)$, fibromas (12\%) and cardiac hemangiomas ( $2.8 \%) .{ }^{1}$ Cardiac rhabdomyoma (CR) range from $1 \mathrm{~mm}$ to $10 \mathrm{~cm}$ in their greatest

${ }^{1}$ Associate Professor, ${ }^{2}$ Assistant Professor, ${ }^{3}$ Professor and Unit Head, ${ }^{4}$ Pathologist, ${ }^{5}$ Professor and Head, ${ }^{6,7}$ Postgraduate Student

1-3,5-7 Department of Obstetrics and Gynecology, PES Institute of Medical Sciences and Research, Kuppam, Andhra Pradesh, India

${ }^{4}$ Sri Path Lab, Bengaluru, Karnataka, India

Corresponding Author: Lingegowda Krishna, Medical Superintendent, Professor and Unit Head, Department of Obstetrics and Gynecology, PES Institute of Medical Sciences and Research Kuppam, Chittoor, Andhra Pradesh, India, Phone: +9391833730 e-mail: drlkrishnas@gmail.com dimension and can be either isolated or multiple. It is usually (50-78\%) associated with tuberous sclerosis complex (TSC). Multiple CR in a neonate with tuberous sclerosis were first described by Von Recklinghausen in 1862. Cardiac rhabdomyomas can occur sporadically (more than 50\%), in association with congenital heart malformations, or in the setting of certain genetic disorders like Down syndrome and basal cell nevus syndrome.

Tuberous sclerosis complex or Bournevilleís disease is a neuroectodermal disorder characterized by the classic triad of mental retardation $(71 \%)$, seizures (78\%) and adenoma sebaceum $(27 \%)$, an autosomal dominant-inherited or sporadically occurring disorder characterized by widespread hamartomas that variably involve the brain, kidneys, heart, skin and other organs. ${ }^{2,3}$ Genetic analysis has identified two disease genes: TSC-1 on chromosome 9q34 encodes for the protein hamartin, and TSC-2 on 16p13 encodes for tuberin. These are tumor suppressor genes that appear to assist in the regulation of growth and differentiation of developing cardiomyocytes by antagonising the mammalian target of rapamycin (mTOR) signaling pathway. ${ }^{4}$

Echocardiography or magnetic resonance imaging (MRI) is usually adequate to facilitate the diagnosis of cardiac tumors. Tumor biopsy, with histological assessment, remains as the gold standard for confirmation of the diagnosis.

Cardiac rhabdomyoma usually appears after 20 weeks of gestation, progressively increases in size up to 32 weeks and later starts regressing. The overall prognosis is dependent on the number, size and location of the lesions as well as the presence or absence of associated anomalies. The manifestations of a cardiac tumor in fetal life include arrhythmia, congestive heart failure, hydrops and stillbirth. Standard obstetrical management is appropriate for uncomplicated cases. Delivery should take place in a tertiary care center where a pediatric cardiologist is available. In postnatal life, cardiac tumors may affect the integrity and function of the adjacent cardiac structures leading to severely compromised blood flow due to inflow or outflow tract obstruction, cyanosis, murmur, respiratory distress, myocardial dysfunction, valvular insufficiency, arrhythmias and sudden death. Prognosis in CR associated with TSC is variable depending on the degree of involvement with $30 \%$ mortality by age 5 and $75 \%$ mortality by age 20 , usually due to central nervous 
system involvement. Mortality has been reported to be even higher in tuberous sclerosis patients with cardiac involvement, reportedly up to $50 \%$ mortality during the first year of life and $90 \%$ mortality by puberty. A definitive diagnosis of TSC as well as careful evaluation of other family members is thus imperative for genetic counseling (Fig. 1). ${ }^{5}$

Knowing the outcome of these tumors is important in making treatment decisions.

\section{CASE REPORT}

We report 34-year-old pregnant woman G2P1L1 who presented to us in established preterm labor with $4 \mathrm{~cm}$ cervical dilatation. Fetal heart rate was $160 / \mathrm{min}$ and cardiotocograph was normal. She progressed normally over 5 hours and delivered a live $2.2 \mathrm{~kg}$ male baby appropriate for gestational age with 8 and 9 Apgar scores at 5 and 10 minutes respectively. The neonate was shifted to mother side and it tolerated breast feeds well. Baby suddenly developed cyanosis and died 24 hours after birth. Thorough physical examination of the neonate did not reveal any cutaneous manifestations of tuberous sclerosis.

Baby's blood was sent for genetic analysis and the baby was sent for autopsy in view of prenatal diagnosis of cardiac rhabdomyoma.

\section{INVESTIGATIONS}

\section{Antenatal}

1. USG: $6 \times 7 \mathrm{~mm}$ of homogenous, hypo-hyper-echoic mass lesion suggestive of rhabdomyoma was observed in the left ventricle at the site of interventricular septum at 24 weeks of gestation (Fig. 2). No extracardiac abnormality was detected. Cardiac functions and rhythm was normal and the lesion increased in size over 3 months to reach the size of $16 \times 11 \mathrm{~mm}$ at 33 weeks of gestation.

2. Genetic counseling: Family pedigree was examined and found no evidence of TSC. Parents declined their tests for mutations of TSC and amniocentesis to diagnose associated tuberous sclerosis in view of associated risks of the procedure.

\section{Postnatal}

1. Fetal autopsy demonstrated cardiac rhabdomyoma. Grossly, rhabdomyoma appeared as white nodule in myocardium.

2. Genetic analysis of neonatal blood revealed no association with TSC.

\section{Diagnosis}

G2P1L1 of 34 weeks of gestation with cephalic presentation in established preterm labor.

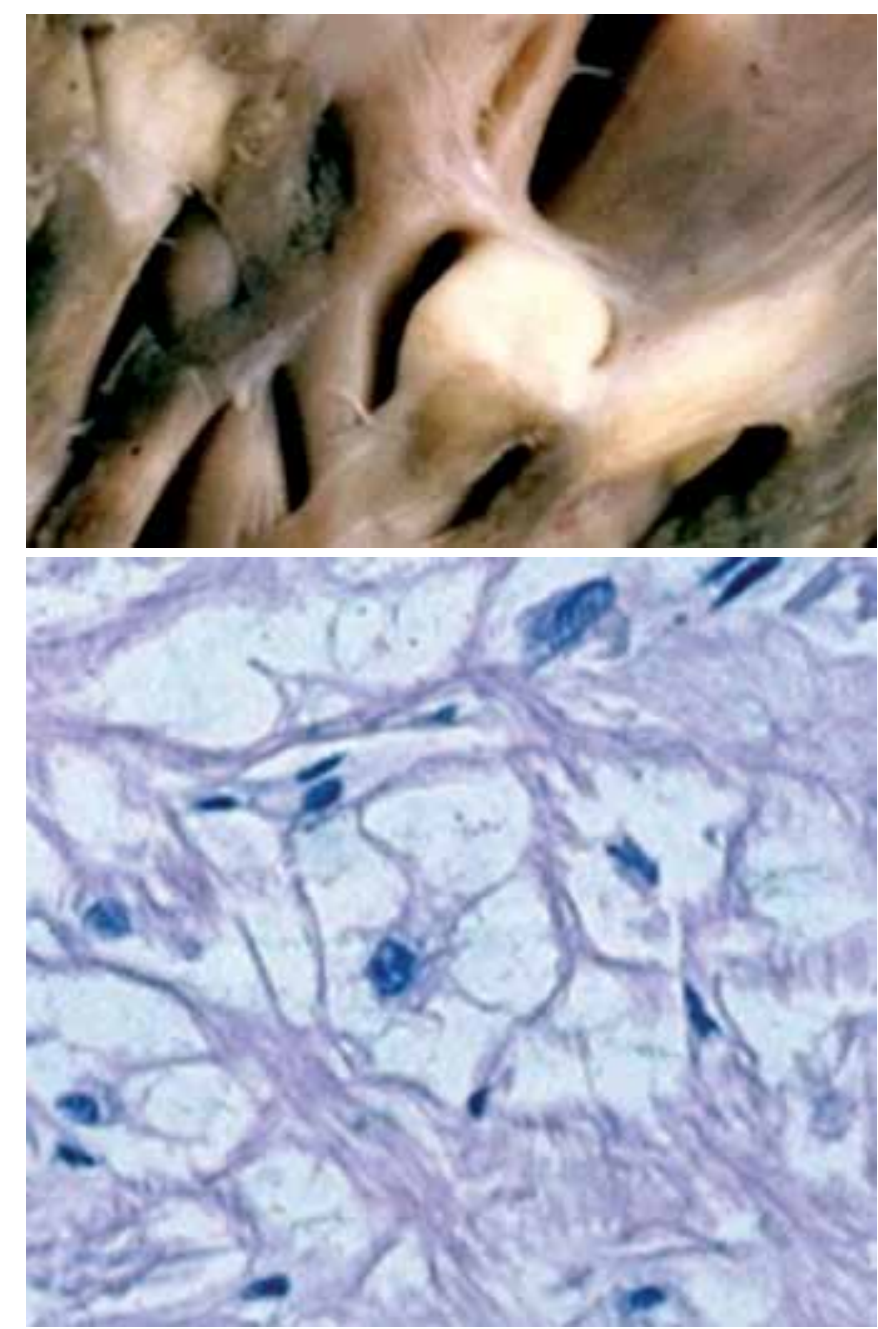

Fig. 1: Microscopy showed typical 'spider cell'

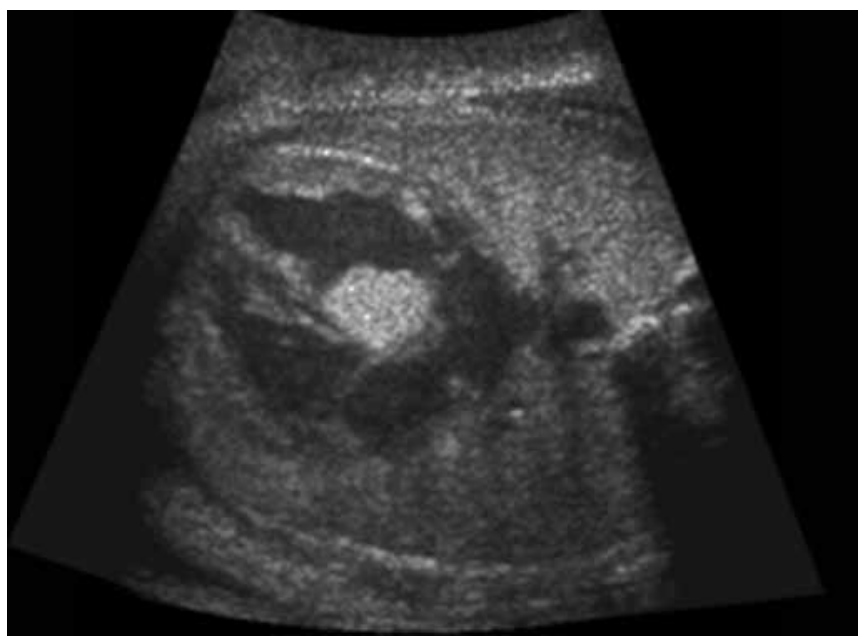

Fig. 2: A single large cardiac rhabdomyoma was seen involving the interventricular septum

The neonate having isolated single cardiac rhabdomyoma with undiagnosed cardiac dysrhythmia resulting in early neonatal death.

\section{DISCUSSION}

The incidence of cardiac tumors is one to two cases per 10,000 live births. ${ }^{6}$ Detection is facilitated by routine 
inclusion of the four-chamber heart view. They occur with equal frequency in the left and right ventricle and the interventricular septum. They are usually associated with TSC in 52 to $86 \%$ of patients with rhabdomyomas. Conversely, $50 \%$ of children with tuberous sclerosis have rhabdomyomas on echocardiographic evaluation. The risk of recurrence of $\mathrm{CR}$ is more in patients with $\mathrm{CR}$ associated with TSC. ${ }^{7}$

Although the association of multiple cardiac rhabdomyomas with tuberous sclerosis has long been recognized, the association with a single rhabdomyoma is not clear. However, in case of a solitary tumor a careful examination of cardiac chambers should be made in order not to miss smaller lesions elsewhere. Multiple cardiac rhabdomyomas in fetal life may herald the diagnosis of tuberous sclerosis well before the other features of the disease, such as skin signs or seizures, emerge in infancy. ${ }^{8}$ The classic clinical triad of tuberous sclerosis includes mental retardation, epilepsy, and facial angiofibromas. The hypopigmented 'mountain ash' macules, angiofibromas, and retinal astrocytic tumors are early features that can occasionally be seen at birth. Other late-occurring cutaneous manifestations of tuberous sclerosis include subcutaneous nodules, subungual fibromas, linear epidermal nevi, and café au lait pigmentation. Seizures during the neonatal period are not infrequent. Imaging may reveal the presence of cortical or subependymal tubers. Renal angiomyolipomas usually manifest later in life. Detection of multiple cardiac tumors should raise a strong suspicion of rhabdomyoma, hence tuberous sclerosis. In such patients, a detailed family history should be obtained and genetic counseling should be offered. ${ }^{9}$

The prenatal diagnosis of solitary rhabdomyoma on ultrasound was confirmed in the index case with the help of biopsy during autopsy of the baby. It was not associated with tuberous sclerosis as was confirmed by karyotyping of the baby.

The outcome of antenatally detected cardiac rhabdomyomas is favorable. Once fetal somatic growth is completed, hamartomas lose their mitotic potential and undergo apoptosis. The reported survival rates range from 81 to $92 \%$. Despite the expected shrinkage of these tumors, unexpected fetal loss or neonatal death may occur due to arrhythmias or obstruction of blood flow ${ }^{10}$ as happened in the present case. After birth, these tumors can be managed conservatively with echocardiography and ECG monitoring. Following birth, tumor regression is a rule rather than an exception. Complete resolution of more than $80 \%$ of the tumors may occur during early childhood. Surgical intervention should be reserved only for sick patients with symptoms of severe obstruction with hemodynamic compromise or hemodynamically significant and intractable arrhythmias that are unresponsive to antiarrhythmic drugs. Operative management involves removal of the intracavitary portion of the tumor without complete excision of the entire lesion. Family screening for tuberous sclerosis is suggested. ${ }^{11}$

\section{CONCLUSION}

Knowledge of the outcome of affected fetuses and the true incidence of tuberous sclerosis in fetal CR is critical for accurate prenatal counseling and planning of prenatal treatment. CR is benign from the cardiovascular standpoint in most affected foeti. Because the natural history of the $\mathrm{CR}$ is one of regression, most patients can be managed conservatively. Finally, it has been suggested that fetuses with a single CR are at lower risk for the development of tuberous sclerosis. ${ }^{12}$

\section{REFERENCES}

1. Chan HS, Sonley MJ, Moes CA, Daneman A, Smith CR, Martin DJ. Primary and secondary tumours of childhood involving the heart, pericardium, and great vessels. A report of 75 cases and review of the literature. Cancer 1985;56:825-836.

2. Lam KY, Dickens P, Chan AC. Tumors of the heart. A 20-year experience with a review of 12,485 consecutive autopsies. Arch Pathol Lab Med 1993;117:1027-1031.

3. Medley BE, McLeod RA, Houser OW. Tuberous sclerosis. Seminars in Roentgenology 1976;11:35.

4. Kotulska K, Larysz-Brysz M, Grajkowska W, Jóźwiak J, Włodarski P, Sahin M, et al. Cardiac rhabdomyomas in tuberous sclerosis complex show apoptosis regulation and mTOR pathway abnormalities. Pediatric and Developmental Pathology March 2009;12(2):89-95.

5. Groves AM, Fagg NL, Cook AC, Allan LD. Cardiac tumours in intrauterine life. Arch Dis Child 1992;67:1189-1192.

6. Mcallister H Jr. Primary tumours of the heart and pericardium. Pathol Annul 1979; 14:325-330.

7. Nir A, Tajik AJ, Freeman WK, Seward JB, Offord KP, Edwards WD, Mair DD, Gomez MR. Tuberous sclerosis and cardiac rhabdomyoma. Am J Cardiol 1995;76:419-421.

8. Becker AE. Primary heart tumors in the pediatric age group: a review of salient pathologic features relevant for clinicians. Pediatr Cardiol 2000;21:317-323.

9. Roach ES, Gomez MR, Northrup H. Tuberous sclerosis complex consensus conference: revised clinical diagnostic criteria. J Child Neurol 1998;13(12):624-628.

10. Krymskaya VP. Tumour suppressors hamartin and tuberin: intracellular signalling. Cell Signal 2003;15(8):729-739.

11. Bosi G, Linthermans JP, Pellegrino PA, Svaluto-Moreolo G, Vliers A. The natural history of cardiac rhabdomyoma with and without tuberous sclerosis. Acta Paediatr 1996;85:928-931.

12. Nir A, Tajik AJ, Freeman WK, Seward JB, Offord KP, Edwards WD, Mair DD, Gomez MR. Tuberous sclerosis and cardiac rhabdomyoma. Am J Cardiol 1995;76:419-421. 